\title{
Kernos
}

Revue internationale et pluridisciplinaire de religion grecque antique

$13 \mid 2000$

Varia

\section{Les devins chez Homère}

Essai d'analyse

\section{Maria Teresa Di Sacco Franco}

\section{(2) OpenEdition}

1 Journals

\section{Édition électronique}

URL : http://journals.openedition.org/kernos/1291

DOI : 10.4000/kernos. 1291

ISSN : 2034-7871

Éditeur

Centre international d'étude de la religion grecque antique

\section{Édition imprimée}

Date de publication : 1 janvier 2000

ISSN : 0776-3824

\section{Référence électronique}

Maria Teresa Di Sacco Franco, «Les devins chez Homère », Kernos [En ligne], 13 | 2000, mis en ligne le 21 avril 2011, consulté le 02 mai 2019. URL : http://journals.openedition.org/kernos/1291 ; DOI :

10.4000/kernos. 1291 
Kermos, 13 (2000), p. 35-46.

\title{
Les devins chez Homère
}

\author{
Essai d'analyse*
}

Par la variété et la fréquence des épisodes touchant à la divination, la lecture des poèmes homériques ne peut que susciter l'intérêt pour ce thème. En effet, le phénomène de la divination chez Homère est caractérisé par la multiplicité des personnages qui, plus ou moins explicitement, sont présentés comme devins. Cette étude s'attachera tout particulièrement à l'analyse des protagonistes épiques clairement définis comme manteis. Il s'agira d'examiner la figure du devin homérique en relevant les caractéristiques qui contribuent à la définir d'une manière cohérente, en dépit des traits apparemment hétérogènes, voire même contradictoires, qui ressortent de la confrontation des personnages. Dans l'Iliade, c'est la figure de Calchas qui retiendra surtout l'attention. Dans l'Odyssée, on étudiera les épisodes où interviennent Théoclymène et Léodès, de même que Tirésias rencontré par Ulysse dans l'Hadès, avant d'en revenir à l'Tliade et aux portraits d'Hélénos et de Polydamas.

Le mantis d'Homère est avant tout un humain, bien qu'il s'agisse d'un mortel tout à fait singulier ${ }^{1}$. Si on laisse provisoirement de côté le cas de Tirésias, Calchas, Théoclymène et Léodès sont des mortels et, par conséquent, sont intégrés dans la société humaine. En effet, ils exercent leur mantosynè à l'intérieur, et en faveur, du groupe dont ils font partie, en dépit de la place singulière qu'ils occupent au sein de la communauté. C'est dans ce sens que les devins épiques peuvent être considérés comme des dèmiourgoi, définition que $\mathrm{J}$.-P. Vernant a donnée du devin grec en général ${ }^{2}$. Les vicissitudes de Calchas, de Théoclymène ou de Léodès montrent que le devin homérique se définit avant tout par une habileté particulière, la maitrise d'un art qui est non seulement le fondement premier de cette figure, mais aussi l'élément qui en fait un véritable prophète au regard d'autres figures apparemment caractérisées comme devins. C'est donc en vertu d'une aptitude particulière que le mantis est sollicité, surtout face à une situation exceptionnelle pouvant

* Cet article est tiré d'un mémoire de fin d'étude présenté à l'Université de Pise durant l'année académique 1996-1997 et intitulé La divinazione e la figura del mantis nei poemi omerici. Faisaient partie du jury les professeurs C. Grottanelli et R. Di Donato.

1 On pense aux nombreux cas qui voient les devins recevoir des attributs normalement dévolus aux dieux.

2 J.-P. Vernant, Parola e segni muti, in Divinazione e razionalità, Turin, 1982, p. 8 sq. [original français Divination et rationalité, Paris, 1974]. 
provoquer la ruine du groupe dont il ne fait pas toujours partie d'une manière durable.

\section{Calchas}

Le premier passage où Calchas apparaît illustre bien cette situation ${ }^{3}$. En effet, c'est à l'occasion d'une des plus graves crises de l'armée achéenne, c'est-à-dire le loimos causé par Apollon, que la présence du devin apparaît indispensable et décisive, comme l'attestent les paroles des interlocuteurs du mantis. C'est Achille, l'antagoniste d'Agamemnon, qui suggère à l'Atride de recourir à la divination afin de connaître la raison de la colère du dieu et le moyen de sauver l'armée achéenne ${ }^{4}$. En outre, le fils de Pélée souligne que rien de ce qui arrive aux hommes n'est le fruit du hasard. La volonté divine est à l'œuvre dans ces signes ${ }^{5}$. Ce trait apparaît constamment dans les poèmes homériques et, comme on le verra, constitue un élément fondamental dans tous les épisodes de divination. L'art divinatoire repose sur cette conviction qu'un dessein céleste est à l'œuvre dans le monde, surtout quand ce qui arrive est extraordinaire. La volonté des dieux d'instaurer une communication avec les mortels est alors patente.

L'intervention mantique de Calchas suit les paroles d'Achille, une fois que ce dernier a donné au devin certaines garanties : avant de dévoiler à tous les raisons de la colère d'Apollon, Calchas cherche la protection de son interlocuteur. L'allusion du fils de Thestor au rapport existant entre le roi et le vilain exprime très clairement la soumission du mantis aux détenteurs du pouvoir ${ }^{6}$. A ce contexte de risque et d'incertitude s'oppose la certitude absolue du prophète et de son interlocuteur principal quant à l'exactitude du theopropion que Calchas est en train de prononcer. En effet, si le mantis affirme sans hésiter qu'il peut annoncer le motif de la fureur divine, Achille, au moment où il accepte d'être le garant de la vaticination du devin, paraît tout à fait sûr de sa maîtrise divinatoire. Il l'exhorte donc à prononcer l'oracle dont il a connaissance ${ }^{7}$, sous-entendant l'efficacité qu'il accorde à la prophétie, laquelle est confirmée par le déroulement de l'épisode et sa conclusion.

L'intervention divinatoire de Calchas ne repose pas sur une tecbnè pratiquée dans l'intention d'acquérir des connaissances. Le devin possède des facultés qui dépassent la simple technique et relèvent davantage d'une faculté divinatoire intuitive. Le devin est entré en communication avec le dieu qu'il interroge et sert d'intermédiaire pour transmettre la volonté divine aux hommes dans un langage qu'ils peuvent comprendre. Le cadre dans lequel

\footnotetext{
Ном., Il., I, 58-130.

Ном., Il., I, 61-67.

5 Ном., Il., I, 63. D'après Achille, les rêves aussi proviennent de Zeus.

6 HoM., Il., I, 85.

7 Ном., Il., I, 86-87.
} 
s'insère le mantis est sacré : son rôle, d'après Achille, est de révéler aux soldats des oracles sous forme de prière ${ }^{8}$. Le recours à l'un des verbes qui évoquent la prière trouve son fondement dans le rapport singulier qui existe entre le devin et le dieu'. Les mots rassurants d'Achille l'attestent, tout en résumant bien les différents aspects qui définissent le mantis dans l'Iliade. Il fait allusion à sa faculté d'instaurer la communication avec un dieu qui est bien disposé à son égard et lui a donné la maîtrise divinatoire, autrement inaccessible. Le texte définit Calchas comme le meilleur des devins, capable de connaître le passé, le présent et l'avenir, en vertu du don qu'Apollon lui a $\operatorname{accordé}^{10}$. Toutefois, la révélation prophétique de Calchas ne fait pas seulement l'objet de l'estime des chefs achéens; ceux-ci craignent les paroles du devin et l'influence qu'elles peuvent exercer sur l'armée. À cet égard, la violence de la réaction d'Agamemnon est significative ${ }^{11}$, d'autant qu'il est désigné par Calchas comme le premier responsable du loimos ${ }^{12}$. Voilà que le devin, d'abord défini comme le meilleur des manteis, devient pour l'Atride un prophète de malheur ${ }^{13}$.

Le personnage de Calchas montre l'ambiguité de la condition du devin chez Homère : il est considéré comme le vecteur indispensable du salut du groupe humain puisqu'il accède à une vérité inconnaissable aux autres, mais il peut également représenter un danger pour les puissants qui voudraient le dominer et tirer profit de ses capacités. Son indépendance n'est garantie que par l'efficacité de ses prophéties et par la protection de la divinité.

Un deuxième épisode montre encore la maîtrise divinatoire de Calchas ${ }^{14}$, mais cette fois dans un cadre technique, par la pratique de l'ornithomancie. Il s'agit d'un flash-back qui permet à Ulysse d'évoquer la réponse de Calchas lors de l'exécution des rites célébrés à la veille du départ vers Troie pour rendre les dieux propices aux Grecs. L'apparition d'un serpent et le massacre des neuf moineaux qu'il perpètre, restés incompris des soldats et de leurs chefs $^{15}$, sont expliqués par Calchas comme un signe positif. Il garde son calme et voit au-delà des apparences : ces oiseaux représentent les années qui verront les Grecs engagés sous les murs de Troie avant la victoire. Par le rappel de cet épisode, Ulysse veut prévenir les tentations de retraite de l'armée grecque et encourager les soldats. Il affirme avoir l'intention de vérifier l'exactitude des propos de Calchas sans avoir l'air de douter de sa

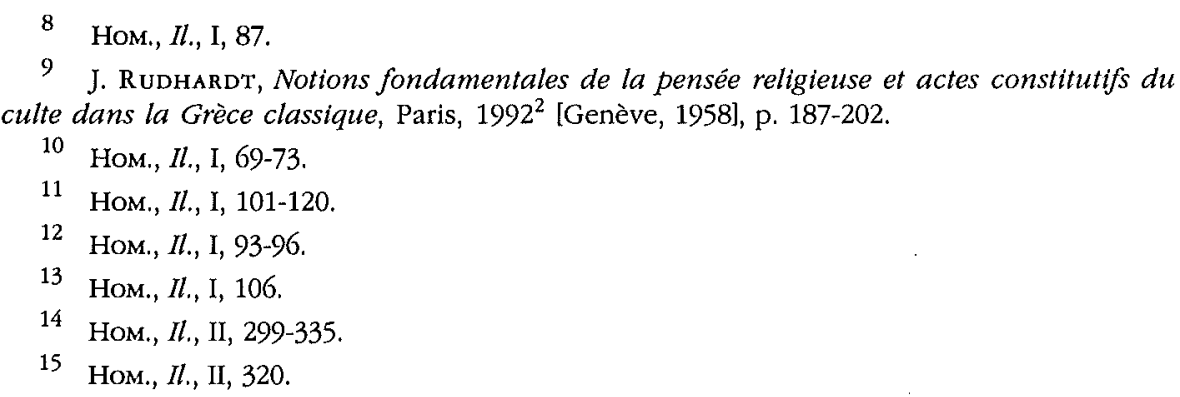


maîtrise divinatoire. On peut donc parler de la valeur symbolique des paroles $\mathrm{du}$ prophète, phénomène que J. Svenbro appelle symbolisation sociale et qu'il attribue en première instance aux aèdes de l'épopée ${ }^{16}$. Les détenteurs du pouvoir cherchent dans les paroles des aèdes, et plus encore dans celles des devins, la confirmation de leurs valeurs et de leurs desseins. Si, dans le premier passage dont on a parlé à propos de Calchas, les mots du devin étaient contraires à la volonté d'Agamemnon, sa prophétie donne ici autorité au plan d'Ulysse. Le mantis présenté dans l'lliade montre donc une maîtrise absolue soit de la divination inspirée, soit de la divination technique. En outre, il est lié aux puissants et aux divinités qui instaurent avec lui une communication. Les dieux cités dans les deux passages sont Apollon et Zeus : on verra en conclusion l'importance de ces divinités quant à la divination homérique.

\section{Théoclymène}

Dans l'Odyssée, le cas de Théoclymène n'est pas tout à fait comparable à celui de Calchas, même s'il présente certaines affinités avec lui. Ce n'est que par une digression dédiée au genos du devin que Théoclymène apparaît ${ }^{17}$. Il pratique la divination pour Télémaque et sa mère, mais il n'est jamais interrogé explicitement comme devin et ses interlocuteurs ne semblent pas être au courant de son habileté divinatoire. Alors que Calchas est le devin attitré de l'armée achéenne, Théoclymène prophétise en faveur d'une communauté à laquelle il est étranger. Il est l'hôte de Télémaque ${ }^{18}$, non son devin. Néanmoins, le phénomène de symbolisation sociale s'applique aussi à ses prophéties : Télémaque et Pénélope accueillent ses paroles avec bienveillance, tandis que les prétendants seront envers lui d'une violence comparable à celle dont Agamemnon fait preuve à l'égard de Calchas ${ }^{19}$. Sur le plan de l'habileté divinatoire, les affinités apparaissent. Tout d'abord, Théoclymène appartient au célèbre genos des Mélampodides, ce qui atteste l'origine apollinienne de son art divinatoire. Cette maîtrise est héréditaire et tous les descendants de Mélampous sont devins ${ }^{20}$. Ensuite, tout comme la prophétie de Calchas à Aulis, le vol du faucon que Théoclymène interprète comme le signe sûr du destin heureux pour le genos d'Ulysse relève d'une habileté ornithomantique.

16 J. Svenbro, La parola e il marmo, Turin, 1984, p. 31-54 Loriginal français La parole et le marbre. Aux origines de la poésie, Lund, 1976].

17 Hom., Od., XV, 222-281.

18 HoM., Od., XV, 508-524

19 SVEnbro, op. cit. (n. 16), p. 31-54.

20 W.R. Hallidax, Greek Divination. A study of its metbods and principles, Chicago, 1967 , p. 79-81. D'après cet auteur, les traditions les plus anciennes sur les devins insistent sur le caractère héréditaire de l'art divinatoire plus que sur l'habileté technique dont il témoigne. 
Le deuxième épisode qui met le devin en scène se situe à Ithaque, après son débarquement du navire de Télémaque. Alors que ce dernier rapporte à sa mère les propos de Ménélas sur les malheurs d'Ulysse révélés au roi de Sparte par le Vieux de la Mer, le devin intervient spontanément. À la certitude du Vieux de la Mer, le Mélampodide oppose la vérité dont il a connaissance : Ulysse se trouve déjà dans sa patrie et il ne tardera pas à se venger de ceux qui aspirent à épouser Pénélope ${ }^{21}$. La conclusion de ce passage présente une légère incohérence puisque Théoclymène affirme avoir déjà dévoilé cet oracle à Télémaque lorsqu'ils se trouvaient près des navires ${ }^{22}$, alors que cette prophétie concernait la descendance d'Ulysse et non un événement particulier. En dépit de sa brièveté, ce passage est très important pour notre propos. Théoclymène est sûr de lui et de l'efficacité de ses prophéties. De ce point de vue, il est proche de Tirésias, dont on parlera plus avant. Cette certitude se fonde sur sa maîtrise divinatoire, mais aussi sur la bienveillance de la reine envers de tels propos, selon le principe de symbolisation sociale mis en avant par J. Svenbro.

Le dernier épisode où Théoclymène apparaît se situe à la cour d'Ulysse ${ }^{23}$ et atteste que son habileté divinatoire repose aussi sur l'inspiration divine et pas seulement sur la technique. En effet, à cette occasion, l'apparition dont Théoclymène est le seul témoin ressemble à une véritable révélation extatique. C'est le seul cas de ce type dans l'épopée. La prophétie n'est pas le fruit d'une interrogation : le devin s'adresse spontanément aux prétendants à la suite du fou rire qu'Athéna a provoqué parmi eux ${ }^{24}$. La vision de Théoclymène suit immédiatement le rire irrépressible des princes. Il paraît à la fois à l'intérieur et à l'extérieur du contexte concret lorsqu'il révèle à ses interlocuteurs la scène de sang et de mort dont il est spectateur en vertu de sa condition de devin ${ }^{25}$. L'opposition constante entre la lumière et l'obscurité qui caractérise le passage permet de parler à l'égard du descendant de Mélampous d'une faculté de voir tout à fait singulière, celle que W.R. Halliday a qualifiée de second sight ${ }^{26}$.

Les prétendants réagissent avec la même violence qu'Agamemnon : la préfiguration de leur défaite cause l'éloignement immédiat de Théoclymène de la cour d'Ulysse ${ }^{27}$. Le devin réagit avec la même tranquillité que Calchas,

21 Hом., Od., XVII, 106-151.

22 Ном., Od., XV, 525-534.

23 Ном., Od., $\mathrm{XX}, 350-373$.

24 Hom., Od., XX, 345-349.

25 C. Grottanel.LI, L'ideologia del banchetto et l'ospite ambiguo, in DdA, n.s. 3 (1981), p. $122-154$.

26 HaLliday, op. cit. (n. 20), p. 75.

27 Ном., Od., XX, 360-362. 
avec la certitude de sa prophétie, mais aussi de la protection divine qui lui est accordée. Les épithètes divines qui lui sont attribuées l'attestent ${ }^{28}$.

\section{Les prophètes et leurs dieux}

Dans les passages considérés, le lien entre les devins et les dieux est souligné tant par l'origine céleste de la mantosynè que par le rôle de ces hommes comme interprètes de la volonté divine. L'origine divine de la faculté divinatoire explique la grande considération dont les prophètes jouissent dans la tradition homérique et implique la singularité de ces mortels à l'intérieur même de la société humaine ${ }^{29}$. Apollon a conféré à ces deux hommes leur maîtrise divinatoire et, en ce qui concerne Calchas, si l'épopée le dit simplement fils de Thestor, des traditions postérieures font d'Apollon son père ${ }^{30}$. Les mots mêmes de l'épopée attestent le lien absolument particulier entre le dieu et le devin ${ }^{31}$. Dans le cas de Théoclymène, c'est le poète aussi qui met l'accent sur le lien entre les deux. De ce point de vue, il est difficile d'affirmer que la digression consacrée au genos dont il fait partie serait un ajout postérieur ${ }^{32}$. Non seulement, le passage n'est pas incohérent par rapport au contexte, mais la mention détaillée des aventures des Mélampodides et de leurs rapports avec Apollon donne la mesure de l'importance que l'épopée attribue à Théoclymène et aux devins en général.

La familiarité des deux devins avec Apollon justifie - on voudrait insister sur ce point - leur faculté de pratiquer une divination non seulement technique, mais aussi inspirée. C'est en vertu de cette habileté que le mantis de l'épopée homérique peut acquérir une connaissance qui comprenne les trois dimensions temporelles ${ }^{33}$ et prononcer des oracles dont le déroulement de l'histoire dévoile l'exactitude et l'efficacité. En dernière instance, le mantis, bien que mortel, présente une disposition singulière à dépasser, au moins sur le plan du savoir, les limites des facultés humaines. On ne peut considérer la mantosynè attestée par les poèmes homériques comme le résultat d'une simple technè fondée sur des correspondances et, par conséquent, comme une technique incertaine. L'incertitude du devin, on l'a vu, tire son origine de l'arrogance des puissants qui, dans certains cas, s'opposent aux vérités prononcées par les prophètes. Ils accentuent en fin de compte le relief du rôle du devin qui ne doute jamais, lui, de sa capacité. Le mantis occupe, dans le cadre de la société aristocratiquement structurée que représente

28 Hom., Od., XV, 271; XVII, 151, XX, 350.

Halliday, op. cit. (n. 20), p. 75 sq.

30 A. Bouché-Leclerce, Histoire de la divination dans l'Antiquité, Paris, 1879-1882, II, p. 39.

31 Hom., $l l .$, I, 69-72.

32 G.S. KIRK, The Songs of Homer, Cambridge, 1962, p. 240 sq.

33 Hом., Il., I, 70. 
l'épopée homérique, une fonction importante: la communauté ne peut renoncer aux devins puisqu'il s'agit d'individus particuliers et non de simples détenteurs d'une technique accessible au premier venu.

\section{Tirésias}

Les traits déjà relevés pour Calchas et Théoclymène sont confirmés dans le cas de Tirésias ${ }^{34}$, en dépit des singularités de ce dernier. Compte tenu de son statut d'âme de l'Hadès, Tirésias est étranger à la société des hommes avec laquelle il ne peut instaurer de lien qu'au travers d'une interrogation nécromantique. Grâce à la prophétie du devin qui se trouve désormais dans l'au-delà, Ulysse se propose de connaître les raisons qui entravent sa navigation et les conditions de son retour à Ithaque. Même dans le moñde des morts, le Thébain n'a pas perdu son habileté divinatoire. L'examen des passages qui le concernent devrait permettre de comprendre quel est le statut du mantis en tant que défunt et, par conséquent, le rapport qu'il entretient soit avec les autres morts, soit avec les vivants.

Les autres habitants de l'Hadès ne peuvent pas acquérir de connaissances sur l'avenir. Même l'âme d'Anticlée, la mère d'Ulysse, qui a conservé le souvenir du passé et connaît les événements présents ${ }^{35}$, ne peut parler de l'avenir. Comme Circé l'a révélé à Ulysse, les âmes ne sont que des ombres voltigeantes, dépourvues de toute faculté cognitive; le héros ne peut s'adresser à elles qu'après avoir sacrifié une victime et offert son sang ${ }^{36}$. C'est le point commun entre l'âme de Tirésias et celle des autres ombres de l'Hadès : le rite préliminaire est indispensable pour s'adresser à lui, mais il a conservé toutes ses facultés, même sa mantosyne $\dot{e}^{37}$. Dès l'instant où il voit son interlocuteur, il le reconnaît, l'appelle par son nom, évoque sa descendance et sa mètis singulière, bien qu'Ulysse n'ait pas dévoilé son identitée ${ }^{38}$. Le devin interroge alors le visiteur sur son voyage dans l'Hadès ${ }^{39}$, mais n'attend pas la réponse et commence à prophétiser en dévoilant ce que le vivant veut apprendre, dans une perspective partiellement différente des prédictions de Circé $^{40}$. La magicienne fille d'Hélios est capable de lui révéler l'avenir, mais Ulysse doit s'adresser au devin pour connaître les raisons qui empêchent le retour dans sa patrie et ce qu'il doit faire pour se rendre les dieux favorables. Tirésias ne prophétise pas dans le détail les vicissitudes qu'Ulysse connaîtra encore, mais insiste plutôt sur la colère de Poseidon et dévoilera même les

\footnotetext{
34 Hом., Od., XI, 90-150.

35 Hом., Od., XI, 155-203.

36 Ном., Od., X, 488-495.

37 Ном., Od., X, 490-493.

38 Ном., Od., XI, 92.

39 Ном., Od., XI, 93-94.

40 Ном., Od., XII, 37-141.
} 
conditions de la mort d'Ulysse ${ }^{41}$. Le mantis se révèle donc capable, comme Calchas, de révéler l'avenir, mais aussi d'éclaircir les causes des problèmes du présent. Cette qualité se fonde sur un rapport privilégié aux immortels, sanctionné par le dieu qui lui a conféré la faculté de prophétiser. De plus, dans le cas de Tirésias, la condition de mort paraît donner à son art une autorité exceptionnelle, qui le met à l'écart des éventuelles menaces de son interlocuteur. Même si la prédiction révèle à Ulysse les difficultés qu'il devra encore traverser, celui-ci écoute attentivement et témoigne d'une grande confiance dans le Thébain. Le sceptre d'or dont le poète parle à l'occasion de la rencontre ${ }^{42}$ et l'épithète d'anax qu'il attribue au devin ${ }^{43}$ sont autant d'éléments significatifs de l'autorité absolue et incontestable des prédictions de Tirésias, sanctionnée par le don exceptionnel de Perséphone qui assure la pérennité post mortem de son $\operatorname{art}^{44}$.

\section{Léodès}

Ce personnage apparemment insignifiant apparaît lors de l'accomplissement de la vengeance d'Ulysse après son retour en Ithaque ${ }^{45}$. En dépit de sa brièveté, ce passage est décisif pour la définition de la figure du mantis chez Homère. Il confirme en outre le rôle joué par le devin dans la société mise en scène par le poète. Partons des mots prononcés par Léodès lorsqu'il supplie Ulysse d'épargner sa vie puisqu'il est étranger aux desseins des prétendants. Il déclare son innocence et se définit comme une victime, des prétendants et d'Ulysse. Pour sa défense, il déclare être soumis aux puissants en tant que thuoskoos ${ }^{46}$. Cet emploi est unique dans l'Odyssée. La réponse du roi souligne lui aussi, mais d'un point de vue opposé, le déséquilibre existant entre le devin et les puissants. Mais en qualité d'haruspice, Léodès ne peut pas, aux yeux d'Ulysse, être étranger aux plans des princes et, par conséquent, doit. être compté au nombre des prétendants. Une fois encore, le détenteur de l'art divinatoire est exploité par ceux dont il dépend.

Le traitement qu'Ulysse entend réserver à trois catégories d'individus résidant en son palais est significatif : Médonte le lâche est épargné par le roi, de même que Phémios l'aède ${ }^{47}$, à la suite des supplications de Télémaque et

\footnotetext{
41 Hom., Od., XI, 134-137.

42 Hom., Od., XI, 91.

43 HoM., Od., XI, 151.

$\begin{array}{ll}45 & \text { Ном., Od., XXII, 310-329. } \\ 46 & \text { Ном., Od., XXII, 318, } 321 . \\ 47 & \text { Ном., Od, XXII, 330-377. }\end{array}$
}

44 L'épopée passe sous silence la généalogie du devin, mais la tradition postérieure le fait descendre de la nymphe Chariclô et attribue sa condition d'aveugle tout comme sa faculté divinatoire à une punition divine. Cf. L. Brisson, Le mythe de Tirésias, Essai d'analyse structurale, Leiden, 1976, p. 25 sq. 
en dépit du fait que, pour Ulysse, le chanteur est complice puisqu'il présente par son art les desseins des puissants. Son rôle social est important, comme l'a remarqué J. Svenbro ${ }^{48}$, mais celui de Léodès doit être considéré davantage encore puisqu'Ulysse décide sa mort, ignore ses supplications et que Télémaque n'intervient pas pour le sauver.

Dans l'Odyssée, Léodès est le seul devin qui appartienne durablement à la communauté pour laquelle il exerce sa mantosynè. Il n'est jamais dit hôte ou étranger, comme c'est le cas pour Théoclymène. Il n'est jamais non plus qualifié de mantis. Il est thuoskoos, terme qui dénote incontestablement la technique divinatoire de la hiéroscopie. Cet emploi dément l'opinion selon laquelle les poèmes homériques n'attesteraient pas la divination fondée sur la lecture et l'exégèse des entrailles des animaux sacrifiés. Un intéressant article d'I. Chirassi Colombo soutient cette opinion et analyse ce prétendu silence comme le signe du.rejet d'une pratique apollinienne jugée incompatible avec l'idéologie de la cité démocratique que refléterait l'épopée ${ }^{49}$. Toutefois, les mésaventures de Léodès semblent témoigner d'une telle pratique, tout comme le dernier chant de l'Iliade. En effet, Priam, avant de rendre visite à Achille pour obtenir le cadavre d'Hector, énumère une série de personnages qui ont un rapport au sacré, les devins en général, les haruspices et les prêtres $^{50}$. Ces allusions ne sont pas fortuites. Elles témoignent de la présence de ce type de divination chez Homère et du lien de cette mantosynè avec Apollon.

\section{Hélénos}

Cette figure apparaît dans l'Iliade à maintes reprises qui montrent son rôle de simple soldat engagé dans la défense de Troie. Toutefois, deux passages mettent en évidence l'ambiguité du rôle du fils de Priam par rapport à la divination. Dans le premier ${ }^{51}$, Hélénos intervient pour exhorter les soldats troyens à résister malgré les adversités de la guerre. À cette occasion, il fait davantage la preuve d'une sorte de sagesse que d'une réelle capacité prophétique. En outre, après s'être ainsi adressé à son frère et à Énée, il donne des instructions à ses camarades afin que les femmes troyennes accomplissent des rites propitiatoires pour Athéna, la divinité qui leur est hostile. En définitive, il montre une inclination pour le sacré, ou mieux pour le culte, tout à fait singulière pour un héros, mais aucun élément sûr ne

48 SVEnBro, op. cit. (n. 16), p. 31 sq.

49 I. Chirassi Colombo, Gli interventi mantici in Omero. Morfologia e funzione della divinazione come modalità di organizzazione del prestigio e del consenso nelle cultura greca arcaica e classica, in M. FALES, C. Grottanellr, Soprannaturale e potere nel mondo antico e nelle società tradizionali, Milan, 1985, p. $145 \mathrm{sq}$.

50 Hом., $I l .$, XXIV, 221.

51 Hoм., Il., VI, 72-102. 
permet de qualifier Hélénos comme devin. Toutefois, une expression du passage en question le présente comme le meilleur parmi ceux qui interprètent les présages ${ }^{52}$. L'absence de toute référence à une divinité qui instaure une communication avec lui ou qui sanctionne son art permet de douter d'une réelle maîtrise divinatoire. L'expression dénote sans doute simplement la sagesse singulière qui caractérise Hélénos parmi les Troyens, en le distinguant de son frère, chef incontestable de l'armée.

La deuxième intervention d'Hélénos suit le dialogue entre Athéna et Apollon à propos du combat imminent entre Hector et Ajax ${ }^{53}$. À cette occasion, le poète attribue à Hélénos la faculté d'entendre les plans des dieux. En effet, il exhorte son frère à combattre, à partir du moment où il a entendu que la mort d'Hector n'est pas proche ${ }^{54}$. De tels propos sont jugés recevables par Hector puisqu'ils sont compatibles avec sa volonté de combattre et de démontrer sa bravoure. En d'autres termes, les mots prononcés par le sage fils de Priam jouent, du point de vue du chef des Troyens, un rôle tout à fait semblable à celui des autres prophéties évoquées jusqu'ici. Toutefois, rien dans ce passage ne permet de définir de manière incontestable Hélénos comme devin. On peut même affirmer qu'il reste singulièrement inactif devant les informations. Il a plutôt l'air d'un messager passif des volontés célestes, le moyen dont se servent les Olympiens pour pousser Hector au combat. Le texte ne dit jamais qu'il interroge un dieu ou interprète un prodige. Or, si l'absence d'interrogation de ce type n'est pas décisive dans le cas d'un devin étranger comme Théoclymène, elle est pour le moins étrange dans le cas d'un individu qui pourrait exercer son art de façon durable dans la société dont il fait partie en tant que fils du roi. De plus, comme on l'a dit, aucune divinité ne vient sanctionner et accréditer sa prétendue habilețé divinatoire. Or tous les autres personnages explicitement qualifiés de devins dans l'épopée reçoivent un tel patronage. Le rôle d'Hélénos chez Homère paraît donc bien loin de celui de devin que la tradition ultérieure lui attribuera de manière incontestable. La condition incertaine d'Hélénos dans l'épopée peut être rapprochée de celle de sa jumelle, qu'Homère entoure d'un silence comparable. Les deux apparitions de Cassandre dans l'Iliade ne la présentent jamais comme prophétesse ${ }^{55}$, contrairement à la tradition postérieure. L'épopée homérique reflète peut-être, sur ces deux personnages, un stade intermédiaire qui n'affirme pas encore la maîtrise divinatoire des enfants de Priam ${ }^{56}$.

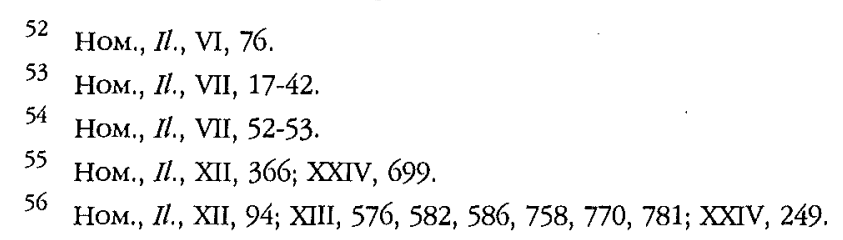




\section{Polydamas}

Les traits de Polydamas sont mieux définis que ceux d'Hélénos et sa figure est moins ambiguë. Toutefois, quelques épisodes ont conduit à lui attribuer un rôle de devin. Le premier passage ${ }^{57}$ voit Polydamas en conseiller d'Hector. Il exprime son opinion en matière de tactique et Hector l'approuve. Le fils de Pantheus n'apparaît donc pas comme un devin, mais comme un combattant de grande importance, proche d'Hector et d'une noble lignée alliée de Priam. Son intervention relève du sage conseil ${ }^{58}$. Le deuxième épisode ${ }^{59}$ présente Polydamas prenant la parole pour dévoiler aux Troyens le message funeste porté par le vol d'un aigle apparu à la gauche de l'armée et tenant dans ses serres un serpent. À cette occasion, il semble le seul des Troyens à comprendre la signification négative du prodige que le texte attribue à Zeus. Toutefois, il n'est jamais qualifié de mantis et cette faculté d'interprétation occasionnelle, en dépit de la confirmation ultérieure de l'exégèse, ne permet pas d'induire une véritable aptitude divinatoire. Et ce d'autant moins que Polydamas lui-même, dans sa réponse à Hector, souligne que la même explication prononcée par un devin aurait reçu du crédit ${ }^{60}$. En effet, Hector a refusé d'accueillir favorablement l'oracle de son ami, arguant du fait qu'il faut se méfier de l'ornithomancie ${ }^{61}$. Cette réaction révèle non seulement la véritable condition de Polydamas, qui est un combattant doué d'une grande sagesse, mais confirme ce que l'on a déjà pu observer au sujet des rapports entre pouvoir et divination. La sagesse de Polydamas est un don divin, tout comme l'excellence d'Hector pour les choses de la guerre ${ }^{62}$. C'est également la volonté divine qui a fait de Calchas, de Théoclymène et de Tirésias des manteis, tandis qu'Hélénos joue un rôle ambigu et que Polydamas est étranger à la divination proprement dite, au-delà de la bienveillance que lui témoignent les dieux.

\section{Conclusions}

L'épopée homérique connaît les deux grands types de divination que l'on qualifie habituellement de " technique ", d'une part, et d'« inspirée », d'autre part. Les véritables manteis maîtrisent l'interprétation des signes, qui constitue le fondement de la divination technique, mais possèdent aussi la faculté extraordinaire de communiquer avec les dieux pour transmettre aux humains leurs messages et leur volonté. Le devin homérique est un intermédiaire entre

\footnotetext{
57 Ном., $I l$., XII, 59-81.

58 Hoм., $l l .$, XII, 80.

59 Hом., Il., XII, 195-250.

60 Hом., Il., XII, 228-229.

61 Hom., Il., XII, 235-240.

62 Hoм., $I l .$, XIII, 730-734.
} 
les hommes et les immortels, dans le but d'accomplir le dessein des divinités. Le devin joue dès lors un rôle précis dans la société homérique. Il s'agit d'un homme qui possède une maîtrise singulière, comme d'autres dèmiourgoi dont parle l'épopée, mais il se trouve dans une position particulière puisqu'il tient entre ses mains le salut de la communauté. Ses performances revêtent une importance inégalée par les autres membres du groupe qui en servent les intérêts. Et c'est précisément ces facultés peu communes qui conduisent les puissants à s'attacher leurs services, tout en n'hésitant pas à blâmer violemment des déclarations gênantes et incontrôlables.

Certains épisodes de divination impliquent des personnages qui ne sont pas des devins et ne concernent que les aspects techniques de cet art, comme l'interprétation des phénomènes célestes, le vol des oiseaux, la clédonomancie ou la cléromancie. Un autre aspect de ces épisodes est l'incertitude qui frappe la prédiction d'individus « profanes » en la matière. C'est donc la mantique inspirée qui constitue la marque incontestable des personnages auxquels l'épopée attribue la véritable condition de devins. De plus, ces devins appartiennent presque toujours à une lignée de manteis et semblent indépendants de tout centre oraculaire et de tout culte officiel dans un sanctuaire. C'est la bienveillance divine qui a accordé cette aptitude à leur lignée et ils en jouissent naturellement, où qu'ils soient.

La poésie homérique témoigne abondamment de la pratique divinatoire, tant technique qu'inspirée, et d'une grande confiance en son efficacité. Ces deux types de divination relèvent de deux divinités différentes, Apollon et Zeus. Tous les épisodes de mantique inspirée font explicitement allusion à Phoibos, tandis que la divination fondée sur la lecture des signes est rapportée au père des dieux, à deux exceptions près, où les interlocuteurs des mortels sont Apollon et Athéna ${ }^{63}$. La poésie homérique distribue donc les compétences entre les dieux pour le patronage de la mantique. Quant aux humains qui bénéficient de cette qualité, ils n'ont pas leur égal parmi les mortels.

Largo Catallo, 1

Maria Teresa Di SAcco Franco

I - 56122 PISA

63 Ном, Il., XV, 526; Od., II, 146-207. 